\title{
Interprofessional teamwork: A case study examining the practice patterns and perceptions of pediatric psychologists
}

Michelle M Ernst*, Stephanie S Filigno, Sandy Cortina, Kelly Byars, Shanna Guilfoyle, Jessica McClure, Beverly Smolyansky, Jessica Kichler and Ahna Pai

Division of Behavioral Medicine and Clinical Psychology, Cincinnati Children's Hospital Medical Center, University of Cincinnati; Hyde Park Child \& Family Therapy, Cincinnati, USA

\begin{abstract}
Interprofessional care including psychologists has been shown to be effective for both primary and subspecialty integrated care. However, providers of behavioral health are infrequently included in studies of interprofessional collaborative practice. The current study explores the activities and perceptions related to interprofessional care of 13 psychologists involved in 19 different interprofessional teams within one institution. To fully explore relevant issues and examine barriers and facilitators to interprofessional teamwork in a systematic and comprehensive fashion, a mixed-method analytic design was used. Quantitative data were collected via survey. Qualitative data obtained by structured interviews were coded for critical themes using grounded theory. Pediatric psychologists working on interprofessional teams were generally satisfied with their experiences. Key facilitators included being valued on the team and using standardized assessments. Barriers centered on financial issues such as scheduling issues impeding billable activity. Based on these findings, we provide a number of recommendations on maximizing clinical care, optimizing the role within the interprofessional team and meeting professional responsibilities.
\end{abstract}

\section{Introduction}

One of the most transformative aspects of the Affordable Care Act (ACA) is the call for an increase in interprofessional collaboration, the process by which care providers from different disciplines collaboratively develop cohesive and comprehensive care plans [1,2]. Relatedly, the American Academy of Pediatrics has endorsed a "medical home" model, highlighting the importance of comprehensive and coordinated care of the "whole child" as well as easy access to services that meet both children's and families' medical and psychosocial needs. This increased commitment to assessing the biopsychosocial needs of the child and family is demonstrated in half of the 26 new ACA-mandated prevention screenings and services related to behavioral health [3]. There is considerable evidence for the value of psychologists in improving pediatric chronic care across a range of populations, service settings, referral concerns and behavioral, functional and biomedical outcomes [4-9]. Research examining the effectiveness of interprofessional care including psychologists has demonstrated improved biopsychosocial outcomes for both primary $[10]$ and subspecialty integrated care $[11,12]$. However, providers of mental and behavioral health are infrequently included in studies of interprofessional collaborative practice [13].

Pediatric psychologists at the current study site had a long history of being involved in interprofessional collaborative practice models with many pediatric subspecialty teams, yet a systematic examination of practice patterns or psychologists' perceptions of these experiences had not been completed. There were several factors fostering the need for a closer examination of our interprofessional collaborative practice processes. First, our institution had recently developed a new strategic plan, in which the delivery of integrated and coordinated care delivery was specifically highlighted as a primary goal. In addition, there had been a greater than 8 -fold increase in the number of psychologists housed in our Division of Behavioral Medicine and Clinical Psychology (BMCP) during the past 15 years (from 8 to 69 psychologists). During this time BMCP had strengthened the presence of behavioral health within the institution through active participation in institutional leadership positions and demonstrating improved patient-reported outcomes, as well as through successful collaborations with other divisions in the medical center. Consequently, the demand for integration of BMCP psychologists into health teams increased. While our interprofessional activities had increased, a careful review of the processes involved in these collaborations had not been completed. It was felt that many psychologists were involved in interprofessional teamwork without a full accounting of the activities or an identification of potential facilitators or barriers. Thus, the objective of this initiative was to conduct a detailed exploration of the practices and issues related to interprofessional collaborative care from the perspective of the BMCP psychologists with a focus on identification of facilitators and barriers. We aimed to subsequently make recommendations to maximize the efficiency and efficacy of interprofessional collaborative practice involving psychologists. Given the relative dearth of research on interprofessionalism that involves behavioral health providers, we also hoped to share our findings with the broader field of interprofessional care.

To fully explore relevant issues and examine barriers and keys to success in a systematic and comprehensive fashion, a mixed-method

Correspondence to: Michelle M Ernst, Division of Behavioral Medicine and Clinical Psychology, Cincinnati Children's Hospital Medical Center, Cincinnati, USA, E-mail: Michelle.Ernst@cchmc.org

Key words: interprofessional care, pediatric psychology, pediatrics, clinical service delivery

Received: September 16, 2016; Accepted: January 16, 2017; Published: October 25,2017 
analytic design was used. Quantitative data were collected via selfreport surveys of the range of services provided as well as psychologist satisfaction with various aspects of care integration, the term used in this study because it aligned with our institution's strategic plan language. Qualitative data of the psychologists' experiences and perspectives of interprofessional teamwork were collected via semistructured interviews and a grounded theory approach [14] was used to elicit key themes, including facilitators and barriers. Of note, at the time the study was conducted, the terminology utilized with the participants and included on the measures was "care integration" because this term was most aligned with the wording of our institution's strategic plan. However, the actual content of our area of inquiry is the work that psychologists do as part of interprofessional teams [1].

\section{Method}

\section{Setting}

Cincinnati Children's Hospital Medical Center (CCHMC) is a pediatric medical center and hospital affiliated with the University of Cincinnati Medical School. At the time of this study, the medical center provided approximately 900,000 outpatient visits, of which the majority were with specialty services. The Division of Behavioral Medicine and Clinical Psychology (BMCP) is housed within the Department of Pediatrics within the University of Cincinnati Medical School. BMCP has demonstrated consistent fiscal stability through clinical fees, insurance reimbursements and grant funding. There is a high demand for BMCP clinical services, so maximizing patient access to services is a priority within BMCP. Of the approximately 70 psychologists who were part of BMCP during the time period of this initiative approximately $75 \%$ primarily did clinical work with the rest primarily grant funded, and approximately half of all psychologists held faculty appointments at the University of Cincinnati. Psychologists whose primary responsibility is to provide clinical service are expected to meet clinical productivity standards (based on a specified number of billed hours per month) which insure high patient access to services and a sustainable financial model for the division. Psychologists' yearly evaluation is based in part on their ability to meet these standards. Thus, psychologists are only able to work collaboratively with medical teams to the extent that they are able to continue to meet their productivity standards.

\section{Data collection}

All interprofessional collaborations in which one specific BMCP psychologist was identified as part of a specific medical team and had worked closely together with team members for at least 1 year to address the biopsychosocial needs of the patients were analyzed. Thus, we chose to only include collaborations which fit the definition of "interprofessional teamwork" in which psychologists were identified as sharing the team identity [1]. Thirteen psychologists fitting this criterion were invited to participate, and all agreed to do so.

For the purposes of quantitative analyses, the unit of analysis was the interprofessional team (IPT). There were 19 separate IPTs in the study involving health care teams that served a range of subspecialty populations including patients with headaches, epidermolysis bullosa, chronic pain, new-onset seizures, neuro-oncologic issues, craniofacial anomalies, sickle cell disease, inflammatory bowel disease, small bowel/ multi-visceral post-transplantation, diabetes, hyper-lipidemia, cystic fibrosis, eosinophilic esophagitis, movement disorders, sleep disorders, conditions requiring neurorehabilitation (e.g., patients with posttraumatic brain injury) as well as patients being considered for small bowel/liver transplant, kidney transplant and bariatric surgery.
The psychologists participating in the study included 13 doctorallevel licensed practitioners. Nine psychologists were part of only one IPT (i.e., integrated with only one medical team), two psychologists were part of 2 different IPTs, and two psychologists were part of 3 different IPTs. An interview and questionnaire were completed for each IPT. Thus, the psychologist was instructed to focus on a specific IPT when providing both the qualitative and quantitative data, and psychologists who were involved in multiple IPTs were interviewed separately for each IPT. Of note, 2 additional psychologists who did not meet the time criteria (i.e., had been involved in their IPT for less than 12 months) were interviewed as well. Their data were not used in analyses but their interview transcripts were used to train coders (see below).

The Institutional Review Board (IRB) was consulted on this study, and determined it as "exempt from review" because the project was undertaken with the goal of applying quality improvement methodology to enhance clinical standards of care. Thus, informed consent for human subjects research was waived provided that no individual information could be identified.

\section{Measures}

Pre-existing measures meeting the aims of this initiative could not be identified in the published literature. Thus, a subset of the study authors reviewed the literature on collaborative care and then used an affinity diagram method [15] based on our own collaborative care experiences and our literature review to identify key domains (e.g "team dynamics", "logistics") that we would want to incorporate into our qualitative and quantitative assessment strategy. These domains were then refined within the larger group of authors which guided development of the survey and interview form that were the assessment tools used for the study.

\section{Quantitative survey (available by request)}

A 13-item questionnaire was developed for the current study to capture psychologists' reports on: 1) care integration activities (including where activities occurred and broken down into activities that occurred during clinic and in clinic space versus activities occurring outside of clinic), 2) patient identification/recruitment processes, 3) administrative processes supporting care integration, 4) financial issues, and 5) location of services provided. Item-level frequencies were calculated for descriptive statistics. Satisfaction levels within each of these areas were assessed using Likert type scaling (1-10) with 10 being the highest level of satisfaction. In addition, two global satisfaction questions related to 1) overall level of care integration and 2) overall administrative processes, using the same Likert scale. Psychologists completed the questionnaire prior to the semi-structured interview.

\section{Qualitative semi-structured interview}

The purpose of the semi-structured interview was to systematically collect information from psychologists about their specific IPT by asking uniform stem questions and using standardized prompts, while offering the flexibility for psychologists to provide additional relevant information and allow the interviewer to ask clarifying questions. Stem-questions included the psychologist-medical team relationship (current status and history), keys to success, barriers to success (including the single most important key and barrier to success), future directions, what the IPT would look like in an ideal world and lessons learned. All interviews were audiotaped and transcribed. 


\section{Procedure}

One project member initially contacted all psychologists who met inclusion criteria by email and explained the purpose of the project as well as requested the opportunity to conduct a 60-90 minute interview. At the time of the interview, the project member reviewed the goals of the interview, answered any questions related to the quantitative survey that psychologists had completed prior to the interview, and conducted the semi-structured interview. The interviewer knew the psychologists as colleagues but was not in a supervisory relationship with any of the them.

\section{Data analysis}

\section{Statistical analysis for quantitative data}

Descriptive statistics were calculated to summarize the data.

\section{Thematic analysis for qualitative data}

Because we were interested in determining new learnings related to care integration from the qualitative data provided in our interviews, our analytic plan was informed by Grounded Theory [14] and interview content was coded using thematic analysis. The thematic analysis started with the process of verbatim transcription of the collected interview data. Psychologists on the study team then repeatedly reviewed transcripts, noted initial themes in the interview content and developed operational definitions for each theme that were tested during weekly meetings with new interview content. Themes were organized under primary domains, and nodes within themes were identified when indicated. This iterative process was repeated until almost all content of the interviews was able to be coded into the identified themes and nodes. Data extracts were organized during two consensus meetings to develop a higher-order framework for the identified themes (available upon request).

After the themes and nodes were identified and operational definitions developed, independent trained research assistants were trained on the transcripts of the psychologists who did not meet inclusion criteria. Because research assistants were not quite at the level of coding that was desired, a $3^{\text {rd }}$ transcript was utilized from our sample of 19 IPTs to continue the training (a transcript taken from a psychologist who had provided two interviews). The research assistants then coded the 18 remaining interviews to classify interview content into relevant themes and nodes (i.e., sub-themes). Five (28\%) of the interviews were coded by both independent coders and demonstrated good inter-rater reliability (80\%). Interview content was classified into a total of 18 themes, some of which were infrequently represented in the data. To focus our analyses, we decided to only include themes which accounted for at least $5 \%$ of total coded content across all interviews in the analysis of facilitator or barriers. All content for these themes were then independently classified by two study team members for valence of the content either being a facilitator or a barrier. Interrater reliability for these valence codes was 0.90 . Chi-square test of independence was conducted to determine if specific themes were more likely a facilitator or barrier to care integration. An alpha of 0.05 was considered statistically significant for all analyses.

\section{Results}

\section{Quantitative data: Care integration activities and satisfaction}

Care integration activities: In a majority of IPTs (72.22\%) psychologists provided at least some services in the medical team's dedicated clinic space. Table 1 depicts the types of psychologist activities done within IPTs depending on whether the psychologist provided service within the clinic space during a time when the medical team was also seeing the patient versus services provided outside of clinic. Fifty percent of IPTs involved psychologists providing services both within clinic space and time as well as outside of clinic. A majority of those IPTs which involved psychological services provided during clinic time and in clinic space included the psychologist consulting with physicians (66.67\%), informally meeting families (61.11\%), attending medical team meetings $(55.56 \%)$ and screening patients $(55.56 \%)$. When IPT psychologist activities were provided outside of clinic time, the most common activities were direct intervention with patients (61.11\%) and medical team meeting attendance (44.44\%). Other IPT psychologist activities (not broken down according to location) included collaborating on research $(44.44 \%)$ and quality improvement initiatives (33.33\%). Patient identification for psychology services differed if completed in the medical clinic versus outside of clinic, with $44.44 \%$ of IPTs that included in-clinic service delivery having the psychologist meeting all patients versus $16.67 \%$ meeting all patients if service delivery was outside of the clinic.

Clinical billings for psychologists' services were the primary means of payment for clinical care in IPTs. Because some of the clinics did not have sufficient patients to support a dedicated psychologist the medical teams financed a portion of the psychologist's non-billable time in $1 / 3$ of the IPTs. In addition, psychologist-supervised trainees provided some unbilled services in $1 / 3$ of the IPTs (one of which was also a IPT which included medical team financial support).

Psychologist satisfaction with interprofessional care: Psychologists generally rated their satisfaction with their IPT quite high. In terms of the two global ratings, the average satisfaction with overall level of care integration was $7.61 \pm 1.50$ out of 10 , with average satisfaction with administrative process somewhat higher $(8.06 \pm 1.39)$. In terms of specific factors, average satisfaction ratings ranged from 7.53 to 9.82 out of 10 , with psychologists most satisfied with the specific administrative processes related to collection of clinical billings, and registration and scheduling of patients. In addition, psychologists were quite satisfied with the manner in which psychology services were introduced to families. Psychologists were least satisfied with areas related to finances and the types of specific IPT activities that they were involved with (rated after psychologists indicated their involvement in the activities listed in Table 1). Examination of open-ended responses to the question of what would increase satisfaction indicated that, in general, psychologists wished they could be involved in more activities within their IPTs.

\section{Qualitative data: Primary themes and key facilitators and barriers}

Primary themes: Content from the interviews were coded into 8 main domains, listed in Table 2 in order of their prevalence. Content related to Medical Team Characteristics was the most prevalent, with $16 \%$ of the total interview content coded within the themes of this domain. Psychologists highlighted that interprofessional collaboration is enhanced when a team values and understands the role of psychology (see facilitators, below). They also noted the importance of appreciating the team culture when joining an interprofessional team.

I think the relationship is key and then going in and listening, observing, and understanding the roles and responsibility and where the power in the team is. That is key. I think too many times people come in with preconceived ideas about what they're going to do - even if you have those back here [in your mind], I think you have to wait and learn 
Ernst MM (2017) Interprofessional teamwork: A case study examining the practice patterns and perceptions of pediatric psychologists

Table 1. In what percent of interprofessional teams do psychologists participate in these types of activities?

\begin{tabular}{|c|c|c|c|}
\hline & & During clinic \& in clinic space & Outside of clinic \\
\hline Direct patient care, non-billable & Meet and greet families (informal) & $61.11 \%$ & $0.00 \%$ \\
\hline \multirow[t]{4}{*}{ Direct patient care, billable } & Screen patients/Triage & $55.56 \%$ & $22.22 \%$ \\
\hline & Formal assessment of patients & $27.78 \%$ & $33.33 \%$ \\
\hline & Intervention with patients & $50.00 \%$ & $61.11 \%$ \\
\hline & Family care conference participation & $16.67 \%$ & $27.28 \%$ \\
\hline \multirow{5}{*}{$\begin{array}{l}\text { Non-direct patient care, non- } \\
\text { billable }\end{array}$} & MD consultation & $66.67 \%$ & $38.89 \%$ \\
\hline & Medical team meeting attendance & $55.56 \%$ & $44.44 \%$ \\
\hline & Psychosocial only team meeting attendance & $11.11 \%$ & $16.67 \%$ \\
\hline & Medical team didactics provision & $16.67 \%$ & $16.67 \%$ \\
\hline & Other & $0.00 \%$ & $5.56 \%$ \\
\hline
\end{tabular}

Table 2. Domains, themes and nodes ( $\mathrm{F}=$ facilitator of interprofessional teamwork; $\mathrm{B}=$ barrier to interprofessional teamwork).

\begin{tabular}{|c|c|c|c|c|c|c|}
\hline Domain & Themes & $\begin{array}{l}\% \text { of Domain-coded } \\
\text { content }\end{array}$ & Node 1 & $\begin{array}{l}\% \text { of Theme- } \\
\text { coded content }\end{array}$ & Node 2 & $\begin{array}{l}\% \text { of Node- coded } \\
\text { content }\end{array}$ \\
\hline \multirow{5}{*}{$\begin{array}{l}\text { Medical Team Characteristics } \\
16.00 \% *\end{array}$} & Value of psychology (F) & $37.90 \%$ & & & & \\
\hline & $\begin{array}{l}\text { Knowledge of psychology } \\
\text { services (F) }\end{array}$ & $18.26 \%$ & & & & \\
\hline & Composition/role definition & $15.98 \%$ & & & & \\
\hline & Shared vision & $13.24 \%$ & & & & \\
\hline & Expectations for psychology & $5.02 \%$ & & & & \\
\hline \multirow{12}{*}{$\begin{array}{l}\text { Psychologist Role } \\
10.88 \%\end{array}$} & \multirow[t]{8}{*}{ Direct patient/family care } & \multirow[t]{8}{*}{$67.10 \%$} & \multirow[t]{4}{*}{ Assess/Screen } & \multirow[t]{4}{*}{$73.00 \%$} & Referral process (F) & $49.32 \%$ \\
\hline & & & & & $\begin{array}{l}\text { Standardized } \\
\text { assessment process (F) }\end{array}$ & $39.73 \%$ \\
\hline & & & & & $\begin{array}{l}\text { Availability of out- } \\
\text { patient care }\end{array}$ & $5.48 \%$ \\
\hline & & & & & $\begin{array}{l}\text { Follow-through with } \\
\text { referrals }\end{array}$ & $5.48 \%$ \\
\hline & & & \multirow[t]{4}{*}{ Intervention } & \multirow[t]{4}{*}{$27.00 \%$} & Specific types & $51.85 \%$ \\
\hline & & & & & Psychoeducation & $25.93 \%$ \\
\hline & & & & & Therapeutic work & $11.11 \%$ \\
\hline & & & & & Availability & $11.11 \%$ \\
\hline & \multirow[t]{3}{*}{ Indirect patient care } & \multirow[t]{3}{*}{$25.50 \%$} & Team education & $47.37 \%$ & & \\
\hline & & & Case management & $28.95 \%$ & & \\
\hline & & & Treatment planning & $21.05 \%$ & & \\
\hline & $\begin{array}{l}\text { Prioritization of team's } \\
\text { patients' needs }\end{array}$ & $7.38 \%$ & & & & \\
\hline \multirow{4}{*}{$\begin{array}{l}\text { Clinic Flow } \\
8.33 \%\end{array}$} & Structure (F) & $60.53 \%$ & & & & \\
\hline & Actual time with patients & $15.79 \%$ & & & & \\
\hline & Scheduling & $16.67 \%$ & & & & \\
\hline & $\begin{array}{l}\text { Identification of high-need } \\
\text { patients to see in clinic }\end{array}$ & $7.02 \%$ & & & & \\
\hline \multirow{5}{*}{$\begin{array}{l}\text { Psychologist Characteristics } \\
7.16 \%\end{array}$} & Experience/expertise (F) & $29.59 \%$ & & & & \\
\hline & Satisfaction (F) & $26.53 \%$ & & & & \\
\hline & Boundaries & $21.43 \%$ & & & & \\
\hline & Transitions & $17.35 \%$ & & & & \\
\hline & Interest/dedication & $5.10 \%$ & & & & \\
\hline \multirow[t]{3}{*}{$\begin{array}{l}\text { Clinical Productivity Standards } \\
6.72 \%\end{array}$} & $\begin{array}{l}\text { Factors out of control of } \\
\text { the psychologist (B) }\end{array}$ & $51.09 \%$ & & & & \\
\hline & $\begin{array}{l}\text { Billable activity } \\
\text { expectations (B) }\end{array}$ & $34.78 \%$ & & & & \\
\hline & Non-billable demands & $14.13 \%$ & & & & \\
\hline \multirow{7}{*}{$\begin{array}{l}\text { Other Financial Factors } \\
5.26 \%\end{array}$} & \multirow[t]{5}{*}{ How services funded } & \multirow[t]{5}{*}{$76.39 \%$} & Trainees & $38.18 \%$ & & \\
\hline & & & Insurance not covering services & $20.00 \%$ & & \\
\hline & & & Medical team contribution & $20.00 \%$ & & \\
\hline & & & Health \& Behavior Codes & $12.73 \%$ & & \\
\hline & & & Grants & $9.09 \%$ & & \\
\hline & Clear business model & $15.28 \%$ & & & & \\
\hline & $\begin{array}{l}\text { Communication of financial } \\
\text { structure to families }\end{array}$ & $6.94 \%$ & & & & \\
\hline \multirow{2}{*}{$\begin{array}{l}\text { Division Factors } \\
5.26 \%\end{array}$} & Resources & $84.72 \%$ & & & & \\
\hline & Communication & $15.28 \%$ & & & & \\
\hline
\end{tabular}

*Percentage of total coded content

Only those domains which accounted for $5 \%$ of total interview content included. 
the culture of the team and how things really go and who's doing what, so that it will be effective.

The Psychologist Role was also relatively prevalent in the content, particularly as related to assessing and screening patients (see facilitators, below). Psychologists also valued their ability to provide direct intervention within the IPT.

I think it has been helpful with some of the cases to be able to provide one-time interventions with them... Although some patients require ongoing services, those one-time interventions seem to work pretty good for the clinic setting.

Psychologists also noted that team education was an essential component of their role in terms of indirectly promoting patient care.

When I started with the group they didn't have much experience with clinical psychology so a lot of what I spent time on was educating them about what role we could play for patients with [chronic illness]. And I actually think there was a real lack of knowledge of what psychologists can do for kids with chronic conditions so for example they didn't realize we could help with things like pill swallowing or behavior management or assessing comorbidities or at least triaging cases so that they were going for assessment to the right places.

Facilitators of interprofessional care: As noted in Table 2, a significantly greater proportion of comments by respondents were classified as facilitators for the following domains: Medical Team Characteristics, Psychologist Role, Clinic Flow and Psychologist Characteristics (all p's<.05). Each of these larger domains was then examined further to identify the specific themes that contributed to these proportional differences. For Medical Team Characteristics, "Value of psychology" and "Knowledge of psychology services" had a greater proportion of comments rated as facilitators. Psychologists spoke about the importance of the medical team valuing what psychology has to offer in terms of psychosocial services to patients as well as how the psychologist can contribute to the overall mission of the team:

What often happens for [the medical team] when [the psychologist] is not there is that the psychosocial comorbidities take up most of the visit. And so for them it is a relief to know that somebody is taking care of the kids more holistically so that they can really focus on the part of their expertise and that they have a second expert coming in to deal with the other issues of the family to help with.

Psychologists also noted that it is critical for the medical team to be well-versed in the psychosocial needs of their patients and how psychologist can intervene in addressing those issues.

Yes, the care model is very structured. The patient comes through with the [medical team] visit and then the Psychology visit. The team knows what we are going to address. They even alert us to things that need to be addressed or work done. There doesn't seem to be any confusion on how to best use our services in the model of that day.

For Psychologist Role "Standardized assessment process" had a greater proportion of comments rated as facilitators, with approaches ranging from use of screeners to more comprehensive approaches. Psychologists noted that these measures assisted both with baseline data collection as well as triaging patients to psychosocial services. "Referral process" also had a greater proportion of comments rated as facilitators, with psychologists commenting on both their own process of referring patients to other providers as well as the process by which patients got referred to them (particularly if they were not co-located).
For Clinic Flow, "Structure", both in terms of the daily flow of the clinic as well as how the psychologist is scheduled to see patients, had a significantly greater proportion of comments rated as facilitators. Psychologists spoke to the issue of being flexible but respectful in terms of getting patients seen by the multiple providers.

Sometimes if we overlap they usually run behind, but I am able to keep on schedule and we kind of work together so if [medical provider] sees someone late I'll go in and see them first and get my part done so then he can see them if he is running behind or the nurse practitioner is going to see them. Actually when I see them we'll come out and we'll talk, we'll staff it in the moment.

Some psychologists also spoke about the value of seeing all of the patients.

It depends on, you know, how many of us are there and all the rest of it, but the idea of the game is that we are going to see all the patients. That was something I set up way early on partly because [the medical team] wanted it and partly because I felt it was really important from the perspective of not stigmatizing people: "You have been selected!" It was just "Oh, we see everybody, here we are".

Finally, for Psychologist Characteristics, "Satisfaction" had a greater proportion of comments rated as facilitators. Psychologists talked about a range of topics, including positive professional relationships and the ability to meet multiple professional goals through the interprofessional collaboration (e.g. both providing clinical service and meeting academic productivity expectations through writing collaborations or conducting research).

It has been my savior in academics actually. I mean I talk about it a lot. It has been a very, very productive journey.

It is just not all formal all the time, so all the personalities that I am interacting with I like, and I like these people, and I think that helps with the coordination of services, and trust and facilitating communication and so on.

Psychologist "Experience/Expertise" also had a greater proportion of comments rated as facilitators, with psychologists commenting in particular on the positive impact specific knowledge of the medical condition had on rapport-building with patients.

I think the biggest thing is being educated about the condition because I came in knowing nothing. The more you learn from the families then when you start talking with a family and using their words - like you have heard from other families in describing things, then you automatically get buy in...I think that's been a key part of the treatment is just being part of the team and understanding the condition.

Barriers to interprofessional care: "Factors Out of Control of the Psychologist" and "Productivity Standards" were the themes noted to have significantly more content rated as a barrier than a facilitator, both within the domain of Clinical Productivity Standards. Psychologists, who typically expressed understanding of the rationale behind having set productivity expectations, also noted the challenge of meeting these expectations in a medical clinic when there is often greater lag time between patients that are ready for the psychologist to see because other providers are seeing the patient. Some psychologists also noted that the unpredictability of the clinic schedule negatively impacted the ability to meet productivity standards.

I think the biggest challenge for us moving forward will be that [the psychologist] numbers are contingent on patients coming in... We don't 
have a lot of control over that part of the process. So what happens on weeks that a nurse practitioner is on vacation, but [the psychologist] is not on vacation and then that can affect her billable hours... So how do you makeup in the deficits where normal things happen? ... And those patients move on and off the schedule so quickly that today you can have four patients on the schedule and tomorrow morning you can have eight patients on the schedule. Or vice versa. You can go from eight to two within a 24 hour period. How are we going to manage that?

Psychologists also noted that there may be activities that are important for clinical care that are not billable, such as informally meeting families or attending interprofessional care conferences.

It's meeting our division's productivity standards concurrent with being in clinic as my medical team needs me to be. And that includes meeting new families where I'm not doing a complete assessment, but I'm just introducing my service and you know sometimes that only takes two to three minutes where you describe it, give a business card and say "let me know". Where other times families - when you say, if you ask "is there anything that is even slightly difficult right now that you think I can be helpful with" and I end up staying in there for 15 minutes.

I think the productivity - I mean it's just in general that I serve that team and whenever they need something like an evaluation or therapy I do it, but I mean I think sometimes it's a barrier that usually my time is really limited so it can only be that hour that I do the evaluation...I do try to go to the other meetings, but without having that leeway, unfortunately, that sometimes it has to be the social worker - like sometimes I will take a few minutes and kind of give her instructions "that is what we talked about, what I did, you know, when you guys have this meeting and talk about that." So I think sometimes that can be a barrier just in terms of that contribution to my productivity is just doing those evaluations and doing therapy.

Psychologists presented ideas for how to address these barriers. For example, the importance of being strategic in using the clinical encounters as a way to also meet other demands, such as building in clinical research, was noted.

If you are going to be spending a lot of time with patients how can you turn that into something that will also allow you to be happy on the academic side of things?

Co-location was also mentioned as a way to maximize efficiency for both patient access and billable activities.

I think we could really be more efficient and get even more families in if we were able to be more present in the clinic with our own space and our own designated time clock.

Psychologists also noted the importance of having open conversations with the medical teams about limitations around providing non-billable services and encouraging other funding models in addition to one based only on the psychologist's billable activities.

I would encourage them to be very forthright, in terms of openly discussing that there is a gray zone of time that doesn't get accounted for. And it needs to be accounted for, so who will account for that?

While meeting clinical productivity standards was noted to be a challenge, many psychologists expressed understanding of the need for a strong financial model.

The expectations are fair. You feel it now, especially like the morning I had today, that you have two new patients scheduled and they both cancel on me. You think how am I going to make up for that? And that feeling is always there, but it comes with the job, it's what you just have to deal with.

\section{Discussion}

This mixed-method analysis of psychologists' perspective on care integration offered insights into psychologists' interprofessional teamwork across a variety of relationships within a single academic medical setting. The quantitative and qualitative findings suggest that, in our institution, psychologists are generally satisfied with many aspects of their interprofessional teamwork and report that they are able to contribute meaningfully to the clinical care of patients.

\section{Activities and satisfaction}

The psychologists interviewed reported a generally high level of satisfaction with their involvement with their IPT. Nearly $3 / 4$ of IPTs had the psychologist co-located with the medical team at least part of the time. In addition, nearly half of the IPTs involved the psychologist meeting all patients during their medical clinic visit. Trainees were involved in several IPTs, which is essential for developing competent clinicians [10] and participants in interprofessional care [16]. Less than $1 / 3$ of the medical teams provided financial support for the psychologist to be part of the IPT, highlighting that psychologists working in interprofessional care settings can develop models in which they are able to be fiscally self-sufficient. However, it is important to note that psychologists described an inability to provide the full range of activities they thought important for clinical care because of limits imposed by a financial model reliant only on billable income.

\section{Facilitators of interprofessional teamwork}

Several of the facilitators identified by the psychologists involved in theIPTs described here were related to the interpersonal and professional functioning of the team. Psychologists identified the team's valuing the contribution of the psychologist to the care of the patient and the functioning of the interprofessional team as a facilitator of the work. The development of trust and respect among team members is thought to be one key relational factor central to successful interprofessional teams [1]. Using realist synthesis of multiple interprofessional studies, Hewitt, Sims \& Harris [17] also identified "support and value" as one of the key mechanisms for interprofessional teamwork. Particularly when psychologists are often "late to the party" in terms of joining already established medical teams, some of which may already have psychosocial providers involved such as social work, it is critical that they get to know the culture of the team and contribute to a mutually respectful working environment. In addition, psychologists are often not the leaders of interprofessional teams serving medical populations, and thus may "serve at the pleasure" of the medical director of the team. These reasons make being able to clearly demonstrate value even more critical.

Relatedly, psychologists indicated that the team's awareness of what psychological services could be provided to the patients was also a facilitator of interprofessional work. Given that a lack of awareness of the services behavioral health providers can offer to medical populations has been identified [18], it is essential that psychologists analyze the composition and functions of other team members in order to clearly outline their own scope of practice and then clearly communicate this to their team. Clear roles within teams help protect against professional boundary infringement and enhance team cooperation [1]. The concept of "working to the top of the license" refers to the ability of team members to identify their discipline's unique contribution to care provision and to focus clinical efforts in that domain [19]. Especially 
when time is limited, psychologists' implementation of a specialized clinical skill set will underscore psychologists' value, foster team's clarity on psychologist scope of practice, and ensure that patients are receiving optimal comprehensive care.

The psychologists involved in IPTs also identified facilitators that were related to direct clinical work with patients. Psychologists highlighted the use of standardized screening for behavioral health issues and appropriate triaging of cases as important to successful IPTs, a role that has been previously identified as critical for both effective care integration and early identification of psychosocial issues compromising health outcomes [20,21]. As experts in the interplay of biopsychosocial factors, this is a unique contribution that pediatric psychologists can make to the interprofessional team's repertoire of patient care tools. Psychologists' experiences and expertise in working with their specific medical population and knowledge of team culture such as language used with and by patients was also noted as an additional IPT facilitator, highlighting the importance of psychologists being familiar with medical conditions when collaborating with medical teams [22]. Also, use of patient language may help all members of the interprofessional team shift from a reliance on the professional jargon of individual disciplines to a common language that can be shared between team members and patients [23].

Clinic processes and structure were also noted as facilitators. In particular, psychologists noted that being a routine part of patient care by seeing all patients served by the interprofessional team promoted universal comprehensive care. In addition, when all patients meet with the mental health provider, even briefly, stigma can be reduced. Previous research suggests routine access to a psychologist is a critical component of integrated care [24], potentially aiding in decreasing stigma associated with mental health services, acceptance of services, and meeting the needs of underserved populations $[25,26]$. Psychologists also noted that flexibility in clinic flow allowed for greater efficiency in patient care. Inefficiencies in clinic management and organization have been noted as barriers to integrated care by other authors as well [20].

Finally, psychologists' satisfaction with IPTs was related to successful IPTs in our study. Our psychologists identified close personal relationships as contributing to their satisfaction. Reeves and colleagues note that both emotional attachments among team members and the use of humor are important relational team processes that underpin interprofessional teamwork [1]. Certainly, caring for patients and families dealing with complex biopsychosocial situations, including end-of-life experiences, can be emotionally draining, and enjoying strong team emotional support can support personal self-care. In addition, psychologists described the benefits of IPT involvement in terms of achieving other important professional goals such as academic productivity.

\section{Barriers to interprofessional teamwork}

Psychologists in the present study highlighted scheduling factors outside of their control as hindering effective clinical care and negatively impacting access for patients to psychological services. In addition, they described that a financial model reliant soley on billable income limited their ability to perform other patient-related and interprofessional activities important for comprehensive care such as having the ability to spend time with all families (even those for whom a billable encounter is not feasible or indicated), or having more time to communicate with team members either informally or at team meetings. These non-billable roles have been identified as essential to being fully integrated into a comprehensive care team [27]. Demonstration of the cost effectiveness of IPT models that make some allowance for unbilled services while optimizing reimbursement of billed care will be imperative for sustained fiscal stability of IPT models [25].

\section{Limitations}

Results presented in the present study possess limitations inherent in qualitative and mixed-methods research. Response bias is possible, in particular because psychologists were reporting directly to an interviewer who was a colleague. The psychologists interviewed were all from one hospital; therefore, the data presented here may not generalize to IPTs in settings with different administrative and financial structures. Although we believe that thematic saturation, the point at which no new themes were being identified, was achieved, it is possible that it was not reached and other themes remain. At the time of the analysis, there were not additional psychologists in the division to interview to determine if additional themes would be identified. Due to the lack of existing measures to assess aspects of the interprofessional teamwork that we were interested in, the questionnaire used to collect the quantitative data was developed specifically for this study, and psychometric qualities of the questionnaire have not been evaluated. Finally, sample size precluded inferential statistical analysis of the quantitative data.

\section{Summary and recommendations}

The findings from the present study underscore that pediatric psychologists can play a valued role in interprofessional teamwork and find satisfaction in doing it, particularly within financial models that support a full range of activities. The findings also highlight a topic that is not frequently discussed in the literature on interprofessional care - the importance of successfully navigating multiple role expectations (e.g., academic productivity) while being engaged in interprofessional teamwork. Based on these findings, we have recommended a standard practice for the development and maintenance of interprofessional collaborative relationships to be used with any interprofessional team seeking behavioral medicine services which ensures implementation of keys to success and which mitigates barriers to success. In particular, we make the following recommendations, and indicate the steps our division has taken to enact these recommendations.

\section{Needs assessment and model proposal for new interprofessional collaborations}

When a medical team requests psychology involvement in an interprofessional team, a standard process for a "needs assessment" process will occur to facilitate identification of an optimal interprofessional team model which integrates the medical team's specific goals, to the extent that they are realistic and achievable, with the expert recommendations from BMCP regarding how to best achieve optimal biopsychosocial outcomes. This needs assessment will include consideration of the patient population biopsychosocial risk as well as the realities of the medical care delivery processes (e.g., clinic space, clinic scheduling practices, other providers' involvement). Then, a care model is developed in which the pediatric psychologist will be able to deliver their unique clinical expertise in a way that best meets the population needs. Once an optimal model is developed, BMCP presents the model to the medical team and other interprofessional team members, including a discussion of the financial support that would be needed to make the model sustainable. The model is then finalized and implemented, with on-going analysis of effective care 
delivery, quality of interprofessional interactions, financial stability and high patient access.

As part of this process, a range of financial support options are explored. Funding through billable activities continues to be encouraged because it supports psychologist autonomy and represents high patient access to services. In addition, medical divisions can contract to provide financial coverage for psychologist activities that are not billable but are deemed essential for optimal clinical care and interprofessional contribution. As an example, when an interprofessional team recently approached our division to inquire about including a psychologist into the care of patients with relatively rare and complex chronic conditions, the division negotiated both financial support from the medical team for the psychologist to be able to attend all team meetings as well as financial support for a "ramp-up" period during which the psychologist was able to get mentoring from and shadow other pediatric psychologist experts in these conditions.

\section{Routine collection of process and clinic outcomes to assess and underscore value of psychology to interprofessional care}

Psychologists' use of standardized assessments can function to identify psychosocial needs in the population, guide intervention and serve as outcome measures. These assessments can be the foundation for regularly scheduled data-driven feedback sessions that psychologists conduct with their interprofessional team to demonstrate both the frequency and quality of behavioral health clinical care. In this way, interprofessional teams become more knowledgeable about psychological services and targets of treatment, and the value of the contribution of psychology is highlighted. In our division, several behavioral health outcome measures are used routinely both within and outside of interprofessional collaborative relationships, some of which are collected electronically $[28,29]$.

\section{Alignment of interprofessional work with other professional goals}

Pediatric psychologists who work in academic medical settings typically have a number of professional responsibilities beyond direct clinical care such as research and training expectations [30]. Effective use of time and resources is essential for successful management of a career, so maximizing the impact of professional activities across role domains is critical. The use of clinical outcome data as noted above can as serve as clinical research which both promotes professional development and achievements as well as contribute to scientific knowledge. Psychologists in our division are encouraged to establish clinical databases early in their interprofessional team involvement in order to meet the clinical and process goals above, but also with the realization of the sample sizes required for successful publication of results. In addition, clinically-focused psychologists are encouraged to meet with more research-focused psychologists who share clinical interests in order to benefit from their expertise.

\section{Streamlining processes, maximizing knowledge and providing emotional support}

This project was initiated due to a realization that our division had a wealth of experience in interprofessional care that we were not accessing to make our work more efficient and provide optimal peer support. The recommendations above have been disseminated within our division. In addition, several communication and support strategies have been initiated. First, we developed a web portal in which written materials that psychologists had developed for their own interprofessional work (e.g., letters to families, marketing materials, team education presentations) were posted and made easily accessible to other psychologists. Second, we initiated a "care integration" presentation at our monthly psychologist staff meeting in which a psychologist involved with interprofessional care briefly presents on their care model including lessons learned. Third, a mentorship group was developed in which more senior psychologists who worked successfully in interprofessional teams mentored more junior psychologists or psychologists newer to interprofessional care.

Successful interprofessional team involvement requires an ability to bring specific skills and knowledge to a team of people collectively working to solve complex biopsychosocial problems. It involves navigating team and personal professional goals while optimally serving the patients and families who are frequently suffering. While these recommendations are focused on promoting successful pediatric psychology involvement in interprofessional teams within one specific institution, we believe that these recommendations have merit for other health care providers working in interprofessional teams.

\section{Declaration of interest}

The authors report no conflicts of interest. The authors alone are responsible for the content and writing of the article.

\section{Acknowledgement}

Dr. Sandy Cortina died unexpectedly as this article was in press. We acknowledge the tremendous psychosocial care she provided to children and families and her unwavering commitment to integrated "whole-child" well-being.

\section{References}

1. Reeves S, Lewin S, Espin S, Zwarenstein M (2011) Interprofessional teamwork for health and social care (Vol. 8): John Wiley \& Sons.

2. Rozensky RH (2012) Health care reform: Preparing the psychology workforce. J Clin Psychol Med Settings 19: 5-11. [Crossref]

3. Tynan WD, Woods KE (2013) Emerging issues: Psychology's place in the primary care pediatric medical home. Clinical Practice in Pediatric Psychology 1: 380-385.

4. Beale IL (2006) Scholarly literature review: Efficacy of psychological interventions for pediatric chronic illnesses. J Pediatr Psychol 31: 437-451. [Crossref]

5. Delamater AM (2009) Psychological care of children and adolescents with diabetes Pediatr Diabetes 10 Suppl 12: 175-184. [Crossref]

6. Ernst MM, Wooldridge JL, Conway E, Dressman K, Weiland J, et al. (2010) Using quality improvement science to implement a multidisciplinary behavioral intervention targeting pediatric inpatient airway clearance. J Pediatr Psychol 35: 14-24. [Crossref]

7. Kashikar-Zuck S, Ting TV, Arnold LM, Bean J, Powers SW, et al. (2012) Cognitive Behavioral therapy for the treatment of juvenile fibromyalgia: A multisite, single-blind, randomized, controlled clinical trial. Arthritis Rheum 64: 297-305. [Crossref]

8. Stark LJ, McGrath A, Janicke DM, Mackner LM, Hommel KA, et al. (2006) A randomized clinical trial of dietary calcium to improve bone accretion in children with juvenile rheumatoid arthritis. J Pediatrics 148: 501-507. [Crossref]

9. Uman LS, Chambers CT, McGrath PJ, Kisely S (2008) A systematic review of randomized controlled trials examining psychological interventions for needle-related procedural pain and distress in children and adolescents: An abbreviated Cochrane review. J Pediatr Psychol 33: 842-854. [Crossref]

10. Runyan CN (2011) Psychology can be indispensable to health care reform and the patient-centered medical home. Psychological Services 8: 53-68.

11. Menon S, Valentini RP, Kapur G, Layfield S, Mattoo TK, et al. (2009) Effectiveness of a multidisciplinary clinic in managing children with chronic kidney disease. Clin J Am Soc Nephrol 4: 1170-1175. [Crossref]

12. Schurman JV, Friesen CA (2010) Integrative treatment approaches: Family satisfaction with a multidisciplinary paediatric abdominal pain clinic. Int J Integr Care 10: 1-9. [Crossref] 
13. Brandt B, Lutfiyya MN, King JA, Chioreso C (2014) A scoping review of interprofessional collaborative practice and education using the lens of the triple aim. $J$ Interprof Care 28: 393-399. [Crossref]

14. Corbin J, Strauss A (2007) Basics of qualitative research: Techniques and procedures for developing grounded theory (3rd ed.). Thousand Oaks, CA: Sage.

15. Simon RW, Canacari EG (2012) A practical guide to applying lean tools and management principles to health care improvement projects. AORN J 95: 85-100. [Crossref]

16. Croker A, Fisher K, Smith T (2014) When students from different professions are colocated: The importance of interprofessional rapport for learning to work together. $J$ Interprof Care 29: 41-48. [Crossref]

17. Hewitt G, Sims S, Harris R (2014) Using realist synthesis to understand the mechanisms of interprofessional teamwork in health and social care. J Interprof Care 28: 501-506. [Crossref]

18. Beacham AO, Herbst A, Streitwieser T, Scheu E, Sieber WJ, et al. (2012) Primary care medical provider attitudes regarding mental health and behavioral medicine in integrated and non-integrated primary care practice settings. J Clin Psychol Med Settings 19: 364-375. [Crossref]

19. Bruns D, Bruns A (2013) The reinvention of the US healthcare systerm: Advocating for a new role for psychology. NYS Psychologist 25: 16-18.

20. Bitar GW, Springer P, Gee R, Graff C, Schydlower M, et al. (2009) Barriers and facilitators of adolescent behavioral health in primary care: Perceptions of primary care providers. Fam Syst Health 27: 346-361. [Crossref]

21. Maddux MH, Bass JA, Geraghty-Sirridge C, Carpenter E, Christenso K, et al. (2013) Assessing psychosocial functioning among youth with newly diagnosed inflammatory bowel disease (IBD): An interdiscplinary clinic approach. Clinical Practice in Pediatric Psychology 1: 333-343.
22. Tovian SM (2006) Interdisciplinary collaboration in outpatient practice. Professional Psychology-Research and Practice 37: 268-272.

23. Hall P (2005) Interprofessional teamwork: Professional cultures as barriers. J Interprof Care 19 Suppl 1: 188-196. [Crossref]

24. Kathol RG, Butler M, Mc Alpine DD, Kane RL (2010) Barriers to physical and mental condition integrated service delivery. Psychosom Med 72: 511-518. [Crossref]

25. Cummings NA, O'Donohue WT, Cummings JL (2009) The financial dimension of integrated behavioral/primary care. J Clin Psychol Med Settings 16: 31-39. [Crossref]

26. Petersen S, Hutchings P, Shrader G, Brake K (2011) Integrating health care: The clear advantage for underserved diverse populations. Psychological Services 8: 69-81.

27. Nash JM, McKay KM, Vogel ME, Masters KS (2012) Functional roles and foundational characteristics of psychologists in integrated primary care. Journal of Clinical Psychology in Medical Settings 19: 93-104. [Crossref]

28. Guilfoyle SM, Follansbee-Junger K, Modi AC (2013) Development and preliminary implementation of a psychosocial service into standard medical care for pediatric epilepsy. Clinical Practice in Pediatric Psychology 1: 276-288.

29. Lynch-Jordan AM, Kashikar-Zuck S, Crosby LE, Lopez WL, Smolyansky BH, et al. (2010) Applying quality improvement methods to implement a measurement system for chronic pain-related disability. J Pediatr Psychol 35: 32-41. [Crossref]

30. Opipari-Arrigan L, Stark L, Drotar D (2006) Benchmarks for work performance of pediatric psychologists. J Pediatr Psychol 31: 630-642. [Crossref]

Copyright: $\odot 2017$ Ernst MM. This is an open-access article distributed under the terms of the Creative Commons Attribution License, which permits unrestricted use, distribution, and reproduction in any medium, provided the original author and source are credited. 